\title{
CircECE1 activates energy metabolism in osteosarcoma by stabilizing c-Myc
}

\author{
Shuying Shen ${ }^{1 \dagger}$, Teng $\mathrm{Yao}^{1+}$, Yining $\mathrm{Xu}^{1}$, Deguang Zhang ${ }^{2}$, Shunwu Fan ${ }^{1 *}$ and Jianjun Ma ${ }^{1 *}$ (D)
}

\begin{abstract}
Background: Osteosarcoma (OS) is the most common malignant bone tumor and has a poor prognosis. The potential involvement of circular RNAs (circRNAs) in OS progression remains unexplored.

Here, we report that CirCECE1, a circular RNA derived from human ECE1, plays a critical role in energy metabolism in OS.

Methods: The RIP chip sequence assay was performed to confirm CircECE1, through overexpression or knockdown of CirCECE1 to verify its function in 143B and U2OS. RNA immunoprecipitation and immunoprecipitation were used to verify CircECE1's regulation of protein c-Myc and CO- immunoprecipitation was used to verified the competitive binding relationship between CirCECE1 and SPOP. The influence of CirCECE1 on energy metabolism was evaluated by seahorse experiment, western blot, and immunohistochemistry.
\end{abstract}

Results: We found that CirCECE1 is highly expressed in OS tissues and cells and that CirCECE1 knockdown suppresses tumor proliferation and metastasis both in vitro and in vivo. Further, CircECE1 significantly promotes glucose metabolism in OS cells in vitro and in vivo. Mechanistically, CircECE1 interacts with c-Myc to prevent speckle-type POZmediated c-Myc ubiquitination and degradation. C-Myc inhibits thioredoxin binding protein (TXNIP) transcription and subsequently activates the Warburg effect.

Conclusions: CircECE1 regulates the Warburg effect through the c-Myc/TXNIP axis. CircECE1 mediated signal transduction plays a important role in OS process and energy metabolism. These findings may identify novel targets for OS molecular therapy.

Keywords: Osteosarcoma, CircECE1, C-Myc, Glucose metabolism, TXNIP

\section{Background}

Although osteosarcoma (OS) is a rare malignancy, it has the second highest incidence and mortality among malignant bone tumors. OS is most common during childhood and adolescence $[1,2]$. It is characterized by direct formation of osteoid tissue and uncontrolled proliferation of bone-related mesenchymal cells and is highly aggressive; $75 \%$ of OS cases have invasion of nearby tissues

\footnotetext{
* Correspondence: shunwufanzjuzju@163.com; sealteam@zju.edu.cn

†Shuying Shen and Teng Yao contributed equally to this work.

'Department of Orthopaedic Surgery, Sir Run Run Shaw Hospital, Medical College of Zhejiang University \& Key Laboratory of Musculoskeletal System Degeneration and Regeneration Translational Research of Zhejiang Province, 3 East Qingchun Road, Hangzhou 310016, Zhejiang Province, China Full list of author information is available at the end of the article
}

$[2,3]$. Although neoadjuvant therapy and wide tumor excision have improved survival, the clinical outcomes and survival rates of OS patients are still unfavorable due to early lung-targeted metastasis. Hence, a better understanding of the biological characteristics and molecular mechanisms of OS carcinogenesis is urgently needed.

With the development of RNA high-throughput sequencing technology and advances in biotechnology, many noncoding RNAs have been found to perform a variety of biological functions in the human body and to participate in the occurrence and development of tumors and other diseases. Circular RNAs (circRNAs), an enigmatic subclass of endogenous long noncoding RNAs that regulate genes at the transcriptional or

C The Author(s). 2020 Open Access This article is licensed under a Creative Commons Attribution 4.0 International License, which permits use, sharing, adaptation, distribution and reproduction in any medium or format, as long as you give appropriate credit to the original author(s) and the source, provide a link to the Creative Commons licence, and indicate if changes were made. The images or other third party material in this article are included in the article's Creative Commons licence, unless indicated otherwise in a credit line to the material. If material is not included in the article's Creative Commons licence and your intended use is not permitted by statutory regulation or exceeds the permitted use, you will need to obtain permission directly from the copyright holder. To view a copy of this licence, visit http://creativecommons.org/licenses/by/4.0/ The Creative Commons Public Domain Dedication waiver (http://creativecommons.org/publicdomain/zero/1.0/) applies to the data made available in this article, unless otherwise stated in a credit line to the data. 
posttranscriptional level, are found in many types of cancer [4-10]. As opposed to linear RNA, circRNA is formed by pre-miRNA through back-splicing of covalently joined 3 ' - and 5 '-ends without the polyadenylated tail. Due to their closed loop structure, circRNAs are able to escape degradation by exonucleases and are much more stable than linear RNAs. Studies have shown that circRNAs serve multiple functions, including acting as miRNA sponges, regulating gene transcription, adsorbing RNA-binding proteins, and regulating protein translation. ${ }^{(8,9)}$ CircRNAs have diverse functions and mechanisms of biogenesis and have been implicated in multiple cancer types. ${ }^{10}$ Our previous study showed that circRNA modulates OS progression [11-13]; however, further research is needed to clarify the functions and roles of circRNA in OS.

Aerobic glycolysis, also termed the Warburg effect, is the most distinguishing difference between normal cells and malignant tumor cells [14-17]. Aerobic glycolysis refers to the preference for glycolysis over oxidative phosphorylation even when oxygen is sufficient. Tumor cells mainly employ the glycolytic pathway to provide energy, and glucose is primarily processed into lactate. Although the efficiency of aerobic glycolysis is unfavorable for energy generation, the ability of tumor cells to evade apoptosis, invade, perform biosynthesis, and resist chemotherapeutics is significantly improved. Additionally, the preference for aerobic glycolysis over oxidative phosphorylation allows cancer cells to adapt and survive when exposed to hypoxic and acidic environments [1820]. Emerging evidence indicates that aberrant metabolism is one of the hallmarks of malignant tumors. The molecular mechanisms underlying the transformation of metabolism are related to activation of oncogenes or loss of tumor suppressors, which ultimately lead to stabilization of hypoxia-inducible factor (HIF) $1 \alpha$ or increased expression of the c-Myc oncogene. The transcription factors HIF $1 \alpha$ and c-Myc promote the expression of glycolytic genes, thus enhancing lactate production and glycolysis [21-23].

Most current circRNA studies focus on the function of circRNAs as endogenous competitive RNAs. Here, we provide the first evidence for a molecular mechanism by which circRNAs enhance the Warburg effect through binding to c-Myc. The results of the current study reveal a new mechanism of osteosarcoma development and demonstrate the significance of the Warburg effect in osteosarcoma.

\section{Methods}

\section{RIP-array assay}

A RIP experiment was used to extract circRNA bound to c-Myc in 143B cells. After the samples were qualified, a Total RNA-seq (H/M/R) Library Prep Kit for Illumina ${ }^{\circ}$ was used to construct the library. First, the designed DNA probe was hybridized with the RNA sample, thereby removing rRNA from the total RNA. Fragmented RNA was used to synthesize the first strand of cDNA. Using a strand-specific method, dUTP was incorporated during synthesis of the second strand of cDNA to label it, and at the same time, end repair was completed in this step. Then, A-tails and ligation adapter were added, ligation products were purified, fragments were sorted according to size, the library was amplified, and the second strand template with dUTP was digested with UDG enzyme before PCR amplification. After amplification, the RIP-Seq library was obtained by purification and recovery with magnetic beads. After the library was constructed, Qubit3.0 was used for preliminary quantification, and then, an Agilent2100 Bioanalyzer was used to detect the size range of the library. When the inserted target fragment size met expectations, Q-PCR was performed to accurately quantify the effective concentration of the library (Library effective concentration $>3 \mathrm{nM}$ ) to ensure the quality of the library. Finally, the circRNA sequences were tested with a Novaseq6000 system using a paired-end 150-bp reading strategy (PE150 mode). The amount of sequencing data was 10G.

\section{Cell culture}

The human cell line hFOB1.19 and the human osteosarcoma cell lines MG-63, U2OS, SJSA-1, HOS, and 143B were purchased from FuHeng Cell Center (Shanghai, China). The OS cell lines were authenticated at ShangHai Biowing Applied Biotechnology Co. Ltd. by performing an STR profiling analysis as described by CapesDavis and according to the ANSI Standard (ASN-0002) set forth by the ATCC Standards Development Organization. Mycoplasma testing was performed using a Venor GeM Mycoplasma Detection Kit (Minerva Biolabs, Berlin, Germany).

\section{Ethics}

All animal experiments were performed according to the Guide for the Care and Use of Laboratory Animals published by the National Institutes of Health and were approved by the Ethics Committee of Sir Run Run Shaw Hospital. All experiments strictly followed the specific guidelines of the panel regarding the care, treatment, and euthanasia of animals used in the study.

\section{Clinical samples}

Slices of formalin-fixed and paraffin-embedded primary osteosarcoma and chondroma tissues were obtained from biopsies of each patient prior to administration of neo-adjuvant chemotherapy. Osteosarcoma and chondroma biopsies were histologically characterized by pathologists according to the criteria defined by the World 
Health Organization. Written informed consent was obtained from each patient prior to study participation, and all study protocols were approved by the Ethics Committee of Sir Run Run Shaw Hospital.

\section{Xenograft tumorigenesis}

Nude mice (4-6 weeks old) were subcutaneously administered $5 \times 10^{6}$ stable $143 \mathrm{~B}$ cells suspended in PBS on one side of the lower dorsal flank ( $n=6$ per group). The width and length of the resulting tumors were measured every week for up to 4 weeks, and tumor volume was calculated according to the following formula: volume $(\mathrm{mm} 3)=($ length $\times$ width2)/2. At 4 weeks postinjection, mice were sacrificed, and tumors were harvested and weighed. Tumor tissues were then used for protein extraction and RNA extraction or fixed for IHC staining.

\section{Tail vein metastasis and bioluminescence imaging}

Cells (143B) were stably transfected with Luc-vector, Luc-circECE1 WT, or Luc- circECE1 MUT. Mice (4-6 weeks old) were then injected with $1 \times 10^{6}$ stable cells via the tail vein. After 4 weeks, mice were anesthetized using isoflurane and intraperitoneally injected with 150 $\mathrm{mg} / \mathrm{kg}$ d-luciferin (Yeason, Shanghai, China). After 15 min, tumors exhibiting luciferase expression were imaged using an IVIS Spectrum in vivo imaging system (Xenogen, Caliper Life Sciences). Images were analyzed using Living Image 4.1 software (Xenogen, Caliper Life Sciences).

\section{Plasmids}

Overexpression vectors for CircECE1 WT or MUT were constructed (BersinBio, Guangzhou, China). The Flagtagged coding sequences of human $\mathrm{c}-\mathrm{Myc}$ and TXNIP were cloned into the lentiviral pDC311-U6-MCMVEGFP vector (purchased from Hanbio Co. Ltd., Shanghai, China) to generate c-Myc and TXNIP expression plasmids.

\section{Cell viability}

Cell viability was determined daily using a CCK-8 kit (Cell Counting Kit-8; Dalian Meilun Biotechnology Co., Ltd) according to the manufacturer's instructions. All observations were reproduced at least six times in independent experiments.

\section{Anoikis assay}

For anoikis analysis, OS cells were cultured in suspension for $48 \mathrm{~h}$. Cells were then trypsinized and stained with Annexin V- APC/7AAD and analyzed by flow cytometry using an Annexin V- APC/7AAD Apoptosis Detection kit (BD Biosciences) according to the manufacturer's instructions. Data were collected on a BD FACSCan and analyzed using the FlowJo software.

\section{Colony formation assay}

OS Cells were seeded in triplicate into 6-well plates at an initial density of 500 cells/well. After 14 days, colonies were fixed with $4 \%$ paraformaldehyde for $20 \mathrm{~min}$ at room temperature $\left(25^{\circ} \mathrm{C}\right)$ and then stained with $5 \mathrm{mg} /$ $\mathrm{ml}$ crystal violet (Sigma). Colonies containing more than 50 OS cells were counted using light microscopy. The average number of colonies was determined from three independent experiments.

\section{RNA extraction and quantitative real-time $P C R$ analysis}

Total cellular RNA was extracted from OS cells or tumor tissues using TRIzol reagent (Invitrogen, Carlsbad, CA, USA) according to the manufacturer's instructions. RNA was stored at $-80^{\circ} \mathrm{C}$. Reverse transcription was performed using $1.0 \mu \mathrm{g}$ total RNA and a HiFiScript cDNA Kit (CWBIO, Beijing, China) to investigate the expression of mRNA. Amplification reactions were performed in $20 \mu \mathrm{l}$ reaction volumes containing amplification primers and UltraSYBR Mixture (with ROX) (CWBIO) that was detected by an ABI 7500 Sequencing Detection System (Applied Biosystems, Foster City, CA, USA). For each amplification reaction, $1 \mu \mathrm{l} \mathrm{cDNA}$ and $1 \mu \mathrm{l}$ primer (Sangon Biotech, Shanghai, China) were used. The cycling conditions were as follows: 40 cycles of denaturation at $95^{\circ} \mathrm{C}$ for $5 \mathrm{~s}$ and amplification at $60^{\circ} \mathrm{C}$ for $24 \mathrm{~s}$. For circRNA, total RNA was incubated with or without $3 \mathrm{U} / \mu \mathrm{g}$ RNase R (Epicentre, San Diego, CA, USA) at $37^{\circ} \mathrm{C}$ for $20 \mathrm{~min}$, and the resulting RNA was subsequently purified using an RNeasy MinElute Cleanup Kit (Qiagen). Specific divergent primers for the back-splice junction of CircECE1 were used to amplify circRNA. The amplified products were detected by agarose gel electrophoresis and sequencing. All reactions were performed in triplicate, and the results were normalized to the mRNA house-keeping gene.

\section{Western blotting}

Following treatment or transfection, culture supernatants were removed, cells or tissues were lysed using radio immunoprecipitation assay buffer (RIPA, Beyotime, China), and protein was harvested and quantified by bicinchoninic acid (BCA) analysis (Beyotime, China). Protein extracts were separated in 10\% SDS-PAGE gels and transferred onto polyvinylidene fluoride (PVDF) membranes (Sigma-Aldrich, USA). After incubation with a high affinity anti-c-Myc antibody (1:1000, Abcam), anti-ELK1 antibody (1:1000, Abcam), anti-cdc25c antibody (1:1000, Abcam), anti-hif1 antibody (1:1000, Abcam), anti-c-Jun antibody (1:1000, Abcam), antiMdm2 antibody (1:1000, Abcam), anti-JunB antibody (1: 1000, Abcam), anti-mct4 antibody (1:1000, Abcam), anti-pdk1 antibody (1:1000, Abcam), anti-pdk4 antibody (1:1000, Abcam), anti-GLUT1 antibody (1:1000, Abcam), 
anti-GLUT4 antibody (1:1000, Abcam), anti-Ubiquitin antibody (1:1000, Abcam) or anti- $\beta$-actin antibody (1: 2000, Cell Signaling Technology, USA) in Primary Antibody Dilution (MB9881, Dalian Meilun Biotechnology Co., Ltd), the membranes were incubated with a secondary antibody (1:5000, Cell Signaling Technology, USA). After washes, signals were detected using FDbio-Femto ECL (Fudebio, Hangzhou, China) and a chemiluminescence system (Bio-Rad, USA). All images were analyzed using Image Lab software.

\section{RNA in situ hybridization}

Cy3-labeled c-Myc probes and Alexa Fluor 488-labeled circECE1 probes were designed and synthesized by RiboBio (Guangzhou, China), and the probes sequences are available upon request. Probe signals were detected using a Fluorescent InSitu Hybridization Kit (RiboBio, Guangzhou, China) according to the manufacturer's instructions. The images were acquired on a Nikon A1Si Laser Scanning Confocal Microscope (Nikon Instruments Inc., Japan).

\section{Glycolysis analysis}

Glucose Uptake Colorimetric Assay Kits (Biovision, USA) and Lactate Colorimetric Assay Kits (Biovision, USA) were used according to the manufacturers' protocols to examine the glycolysis process in osteosarcoma. A Seahorse XF Cell Mitostress test kit and Bioscience XF96 Extracellular Flux Analyzer were used to measure the oxygen consumption rate (OCR) and extracellular acidification rate (ECAR). Briefly, $4 \times 104$ OS cells were seeded into 96-well plates and then incubated overnight. After the cells were washed with Seahorse buffer, oligomycin, FCCP, and rotenone were automatically injected to measure the OCR. Then, glucose, oligomycin, and 2deoxy-glucose (2-DG) were added to measure the ECAR. The OCR and ECAR values were calculated after normalization to the OS cell number and are plotted as the mean \pm SD.

\section{Measurement of mitochondrial membrane potential}

The alteration in the mitochondrial membrane potential $(\triangle \Psi \mathrm{m})$ was measured using a JC-1 kit (Beyotime Biotechnologies, Jiangsu, China). Briefly, U2OS and 143B cells were harvested by scraping and then resuspended in $0.5 \mathrm{~mL}$ of culture medium. Next, $0.5 \mathrm{~mL}$ of JC-1 Staining Solution was added to the cell suspension. Then, the suspension was incubated for $25 \mathrm{~min}$ at $37^{\circ} \mathrm{C}$ in a $\mathrm{CO}_{2}$ incubator. Next, the OS cells were collected by centrifugation at $300 \times \mathrm{g}$ for $10 \mathrm{~min}$ and washed twice with JC-1 Staining Buffer. Subsequently, $500 \mu \mathrm{L}$ of JC-1 Staining Buffer was added to each tube, and the OS cells were resuspended. The samples were then analyzed by flow cytometry. Mitochondrial depolarization was measured by a downregulation in the red/green fluorescence intensity ratio.

\section{Chromatin immunoprecipitation (ChIP) assay}

ChIP assays were performed using an EZ-ChIP kit from Millipore according to the manufacturer's protocol. The primers used to detect TXNIP promoter occupancy were F: 5' -CAGAGCGCAACAACCATT-3' and R: 5' -AGGC TCGTGCTGCCCTCGTGCAC-3'.

\section{Statistical analysis}

Statistical analyses were performed using 22.0 SPSS software. The data are presented as the means with SDs, and statistical significance was determined using unpaired Student's t-tests. The data were analyzed using paired or unpaired t-tests or ANOVA, as appropriate. A $P$-value of $<0.05$ was considered to be statistically significant.

\section{Results \\ CircECE1 is overexpressed in OS tissues and cell lines and is predominantly localized in the cytoplasm}

The RNA immunoprecipitation (RIP) microarray expression profile for detecting circRNAs binding c-Myc in OS cell lines is described in Fig. 1a. We chose 10 circRNAs (input $>0.2$, fold change $>10$, and $\mathrm{fdr}<0.05$ ) to verify binding to c-Myc in 143B cells and found that hsa_circ_ 0002402, or CircECE1, was most significantly enriched (up to 25.13-fold, Supplementary Fig. 1). To investigate the expression of CircECE1 in clinical samples, we collected 10 chondroma and 10 OS samples and performed qRT-PCR to detect CircECE1 expression. CircECE1 was more highly expressed in OS tissue than in chondroma tissue (Fig. 1b). Further, the expression of CircECE1 was higher in pulmonary metastases than in primary OS tissues (Fig. 1c). The expression of CircECE1 was also higher in OS cell lines (HOS, 143B, U2OS, SJSA-1, and MG-63) than in the hFOB1.19 cell line. Among the OS cell lines, U2OS and 143B cells had the highest expression of CircECE1 (Fig. 1d). CircECE1 is generated from the $E C E 1$ gene located on chromosome 1 . The annotation for circECE1 includes exons 2, 3, and 4 of ECE1 (total $442 \mathrm{bp}$ ) (Fig. 1e). Therefore, we assessed the head-to-tail splicing of endogenous CircECE1 by RT-PCR with convergent and divergent primers. The divergent primers for CircECE1, but not GAPDH, amplified a PCR product, indicating a circular and not linear form (Fig. 1f). Similarly, qPCR analysis of total RNA after RNase R treatment indicated that CircECE1 was resistant, whereas ECE1 mRNA transcripts decreased sharply under RNase $\mathrm{R}$ treatment (Fig. 1g). RNA FISH revealed that CircECE1 was mainly localized in the cytoplasm and nucleus (Fig. 1). Taken together, these findings reveal that CircECE1 is overexpressed in OS 
a

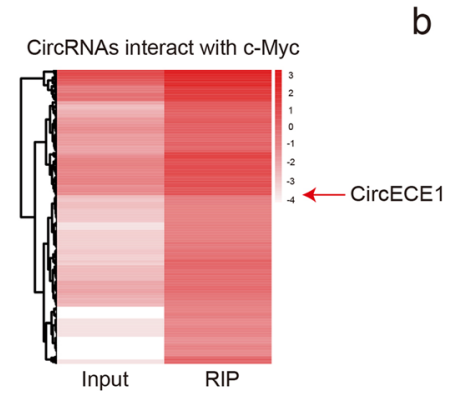

d

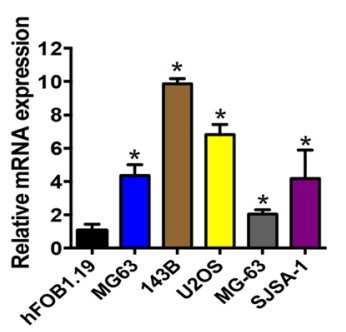

f

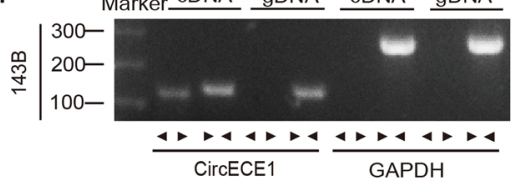

Marker CDNA gDNA CDNA gDNA

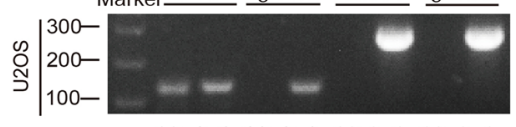

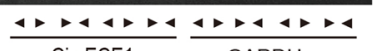

g

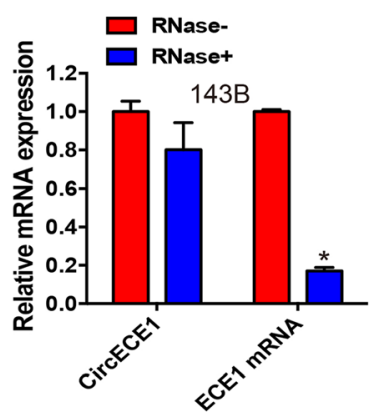

e
C

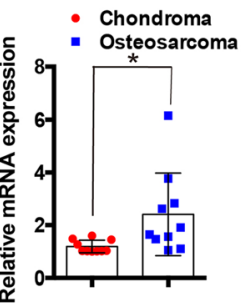

primary OS pulmonary metastasis
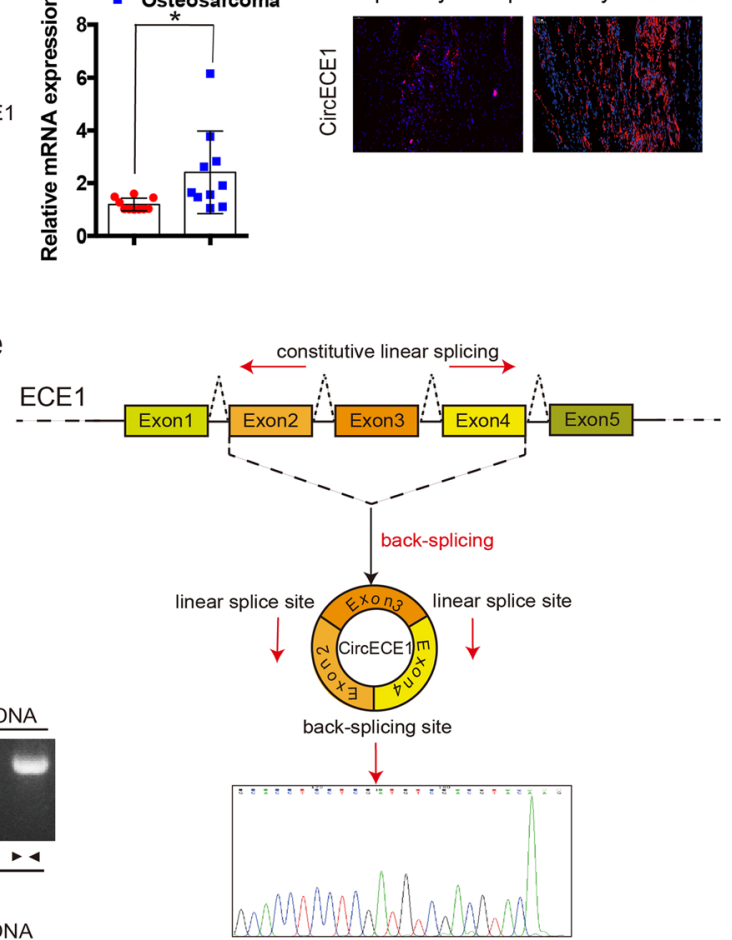

h

CircECE1

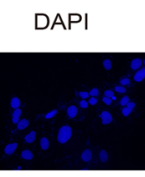

Merge

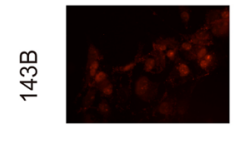

岀
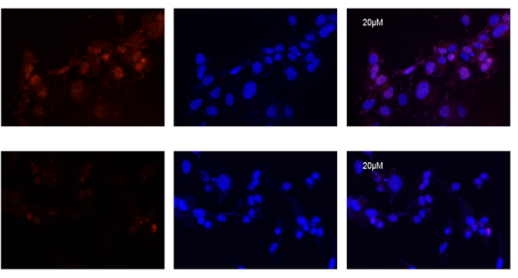

क

O
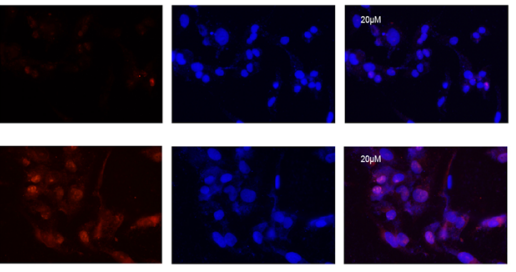

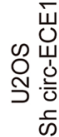
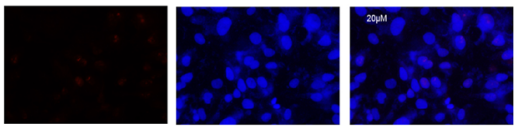

Fig. 1 CircECE1 validation and expression in osteosarcoma tissue and cells. a. RIP-seq results indicate that CircRNAs interact with c-Myc. The red arrow indicates CircECE1. b. Total RNAs were isolated from the specimens of patients with OS and from chondroma tissues for use in real-time PCR. Tumor samples exhibited significantly higher levels of CircECE1 compared to those in the chondroma tissue. Data represent the mean \pm SD $(n=10)$. c. CircECE1 expression was higher in human OS pulmonary metastasis than in primary OS tissues. Representative images are provided (400× magnification). d. CircECE1 expression in hFOB1.19 and osteosarcoma (OS) cell lines (OS-732, 143B, HOS, SJSA-1, and MG-63) was evaluated by qRT-PCR. Data represent the mean \pm standard deviation (SD) $(n=3) .{ }^{*} P<0.05$. e. Schematic illustration reveals the ECE1 exon $2-4$ circularization that forms CircECE1 (red arrow). The presence of CircECE1 was validated by RT-PCR and subsequent Sanger sequencing. The red arrow represents "head-to-tail" CircECE1 splicing sites. f. The presence of CircECE1 was validated in U2OS and 143B osteosarcoma cell lines by RTPCR. Divergent primers amplified circECE1 in CDNA but not in genomic DNA. GAPDH was used as a negative control. $\mathbf{g}$. The expression of CircECE1 and ECE1 mRNA in 143B cells treated with or without RNase $R$ was detected by real-time PCR. The relative levels of CircECE1 and ECE1 mRNA were normalized to the value measured in the mock treatment. Data represent the mean $\pm S D(n=3)$. ${ }^{*} P<0.05$. $\mathbf{h}$. RNA fluorescence in situ hybridization (FISH) revealed that CircECE1 was predominantly localized within the cytoplasm. CircECE1 probes were labeled with Alexa Fluor 488, and nuclei were stained with DAPI. Scale bar $=50 \mu \mathrm{m}$ 
tissues and cell lines and is localized in the cytoplasm and nucleus.

\section{CircECE1 promotes the migration and proliferation of OS cells}

We designed three CircECE1 siRNAs that specifically targeted the junction sites of CircECE1, transfected U2OS and 143B cells with these siRNAs, and then assessed the knockdown efficiency by qRT-PCR. Knockdown experiments with independent small siRNAs designed against back-splicing between exons 2 and 4 of CircECE1 (Fig. S2a) confirmed that these siRNAs selectively targeted CircECE1, and siRNA\#2 provided the most effective knockdown. We then constructed CircECE1 small hairpin RNA (shRNA) using the sequence of siRNA\#2 to stably knockdown the expression of CircECE1 in OS cells (Fig. S2b). We also overexpressed CircECE1 and found that it did not affect ECE1 mRNA levels (Fig. S2c). Cell viability was further evaluated with CCK-8 and EDU assays, which indicated that CircECE1 knockdown impaired proliferation and CircECE1 overexpression promoted proliferation (Fig. 2a-b). Overexpression of CircECE1 also significantly promoted the colonyforming ability of OS cells (Fig. 2c). Soft agar assay results also showed that CircECE1 overexpression increased colony formation (Fig. 2d). Moreover, CircECE1 knockdown augmented the apoptosis rate (Fig. 2e), and anoikis in OS cells could be rescued by CircECE1 overexpression (Fig. 2f). The migration ability of OS cells was prominently decreased by CircECE1 shRNA and increased by CircECE1 overexpression (Fig. 2g). Taken together, these findings reveal the role of CircECE1 in the motility, survival, and proliferation of OS cells in vitro.

\section{CircECE1 regulates c-Myc function. C-Myc regulate the expression of many different genes involved in tumorigenesis}

To determine how CircECE1 affects cancer cell behavior, we determined whether CircECE1 could affect c-Myc function target genes. We conducted RT-qPCR and WB and analyzed the expression of some known c-Myc targets (JunB, Mdm2, HIF1 $\alpha$, c-Jun, ELK-1, and Cdc25a) in our CircECE1-overexpressing and knockdown cells and found that several c-Myc targets were upregulated in the CircECE1-overexpressing OS cells and downregulated in CircECE1 knockdown OS cells (Fig. 2h and i). To validate the effect of CircECE1 in mediating c-Myc function, we investigated whether c-Myc knockdown could reverse the changes in target genes induced by CircECE1 overexpression. c-Myc knockdown blocked the upregulation of JunB, Mdm2, HIF1 $\alpha$, c-Jun, ELK-1, and Cdc25a that was induced by CircECE1 overexpression (Fig. 2i). These results indicate that CircECE1 regulates c-Myc function.
CircECE1 interacts with c-Myc to prevent its degradation by speckle-type POZ (SPOP)

We used two bioinformatic websites (catRAPID and CISBP-RNA) to predict the binding sites of CircECE1 and c-Myc (Fig. S3a-b). To identify the c-Myc regions that interact with CircECE1 in vitro, HEK-293 T cells were cotransfected with CircECE1 and vectors encoding various fragments of the c-Myc protein (Fig. 3a). Our results demonstrated that c-Myc fragment 2 interacted with CircECE1 (Fig. 3b), which matched the predicted binding sites. Next, a series of CircECE1 truncations were constructed to map the CircECE1 fragment that interacts with c-Myc (Fig. S3c). RIP results indicated that truncation 2 and 4 of CircECE1 interacts with c-Myc (Appendix Fig. S3D). Furthermore, we mutated four predicted binding sites in the truncation 2 (100-130 bp) and 4 (301-442 bp) region of CircECE1 to determine whether this mutated form still interacted with c-Myc (Fig. S3e). We found that WT CircECE1, but not MUT CircECE1, interacted with c-Myc in 143B cells (Fig. 3b-c \& Fig. S3f).

Additionally, we found that CircECE1 overexpression resulted in increased levels of $\mathrm{c}-\mathrm{Myc}$ protein in U2OS and 143B cells (Fig. 3d and e) but not increased mRNA levels (Fig. 3f). Given these observations, we hypothesized that CircECE1 regulates c-Myc levels in OS cells posttranscriptionally. To assess this possibility, CircECE1-WT/MUT-overexpressing cells were treated with the protein synthesis inhibitor CHX and CircECE1 knockdown cells were treated with the proteasome inhibitor Bortezomib. CHX treatment decreased c-Myc protein in 143B cells (Fig. 3g). The half-life of c-Myc was significantly longer in CircECE1 WT cells than in control or CircECE1 MUT cells (Fig. 3g). Additionally, Bortezomib treatment reversed the decrease in $\mathrm{c}-\mathrm{Myc}$ protein induced by CircECE1 knockdown (Fig. 3h). IP results also indicated that cMyc was more ubiquitinated in CircECE1 knockdown cells and less ubiquitinated in CircECE1-overexpressing cells compared to control cells after treatment with NEM (Inhibitors of endogenous deubiquitinating enzymes) or PR-619 (Nonselective deubiquitinating enzyme inhibitor) (Fig. 3i-j). These results indicate that CircECE1 regulates c-Myc levels in OS cells posttranscriptionally.

The E3 ubiquitin ligase adapter SPOP has been reported to promote $\mathrm{c}-\mathrm{Myc}$ ubiquitination and degradation. To examine whether SPOP is involved in CircECE1-mediated changes in c-Myc expression, SPOP was knocked down in CircECE1 knockdown U2OS and $143 \mathrm{~B}$ cells. SPOP knockdown abolished the decrease in c-Myc protein levels induced by CircECE1 knockdown (Fig. 3k). Previous reports have shown that SPOP substrates share an SPOP-binding consensus motif, $\phi \pi \mathrm{S} \mathrm{S} /$ 
a

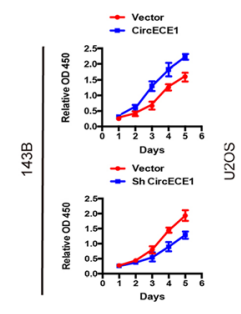

C
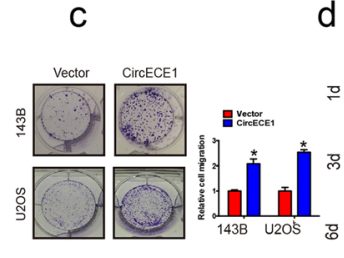

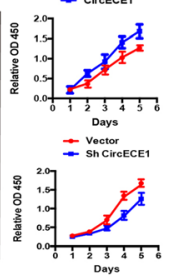

d

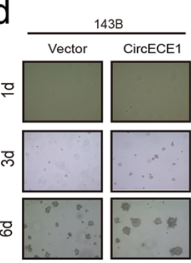

b
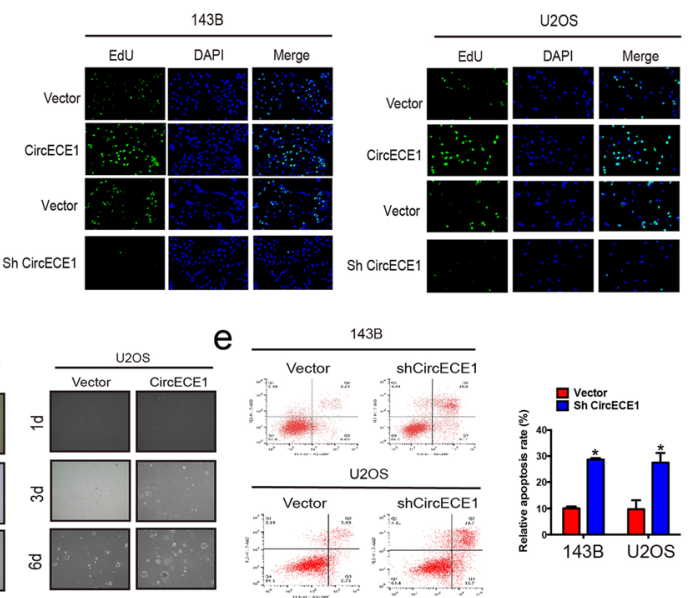

e
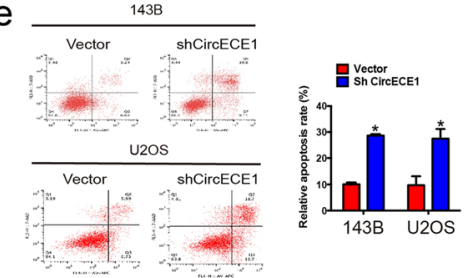
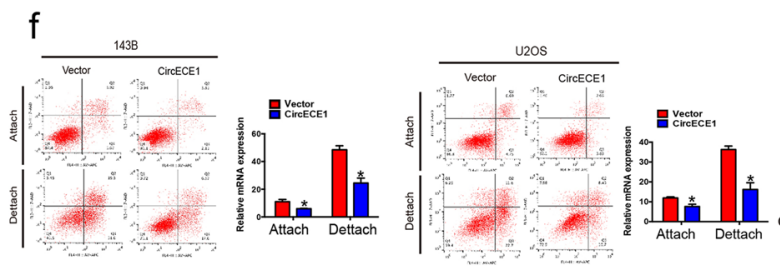

h

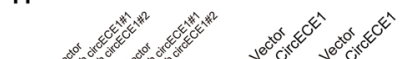

C-M

-

Cdc25A -andc25A
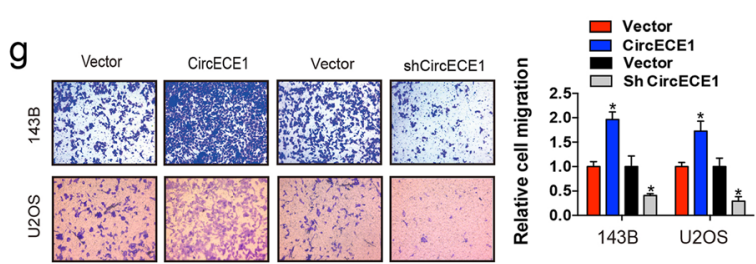

ELK1

JunB

Mdm2

HIF1a $\cdots$

$\beta-a c$

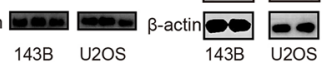

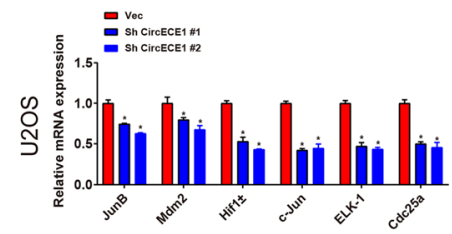

ㅂ.ᅳ.
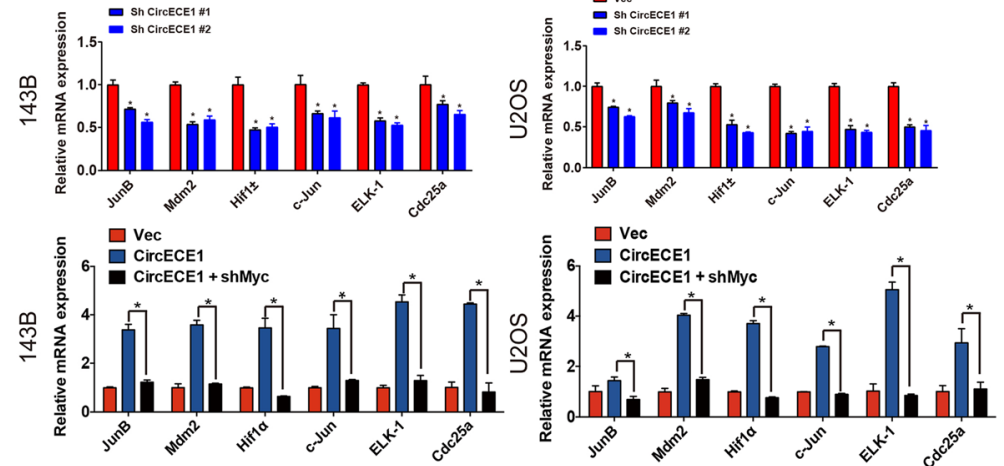

Fig. 2 CircECE1 increased cell survival and colony formation and promoted the expression of c-Myc targets. a. Silencing or overexpression of CircECE1 in osteosarcoma cells decreased or increased, respectively, the ability of cell proliferation compared to that of the control. ${ }^{* *} P<0.01$. Data represent the mean \pm SD $(n=6)$. b. CircECE1 functions in tumor cell proliferation as detected by EdU assay. Nuclei were stained with DAPI, and a combined reaction involving EdU and DAPI indicated the cells in S phase. c. CircECE1 overexpression promotes cell growth as determined by colony formation assay (details are shown in the insets). Error bars represent the mean \pm SD of three independent experiments. ${ }^{*} \mathrm{P}<0.05$. $\mathbf{d}$. Cellular transformation was induced by CircECE1 overexpression. Vector or CircECE1-overexpressing OS stable cells were cultured in soft agar for 20 days. Colonies were stained with crystal violet, photographed, and quantified using ImageJ (details are shown in inserts). e. CircECE1

downregulation in osteosarcoma cells increased cell apoptosis $(n=3)$. $\mathbf{f}$. CircECE1 overexpression in osteosarcoma cells decreased cell anoikis $(n=$ 3). g. Cell migration abilities of U2OS and 143B cells transfected with CircECE1 or vector were evaluated by transwell migration assays. Data represent the mean $\pm S D(n=3)$. ${ }^{*} P<0.05$. Scale bar, $50 \mu \mathrm{m}$. $\mathbf{h}$. Protein lysates from vector and CircECE1 or shCircECE1-transfected osteosarcoma cells were subject to western blotting. Cell lysates were analyzed using c-Myc, HIF-1a, Cdc25a, Mdm2, ELK1, JUN, and JUNB antibodies. i. The shCircECE1 or CircECE1 and the vector transfected osteosarcoma cells were maintained at $80 \%$ confluence and were used for RNA extraction and measurement of c-Myc and c-Myc targets. The CircECE1 cells expressed significantly higher levels of c-Myc targets compared to those of the control 


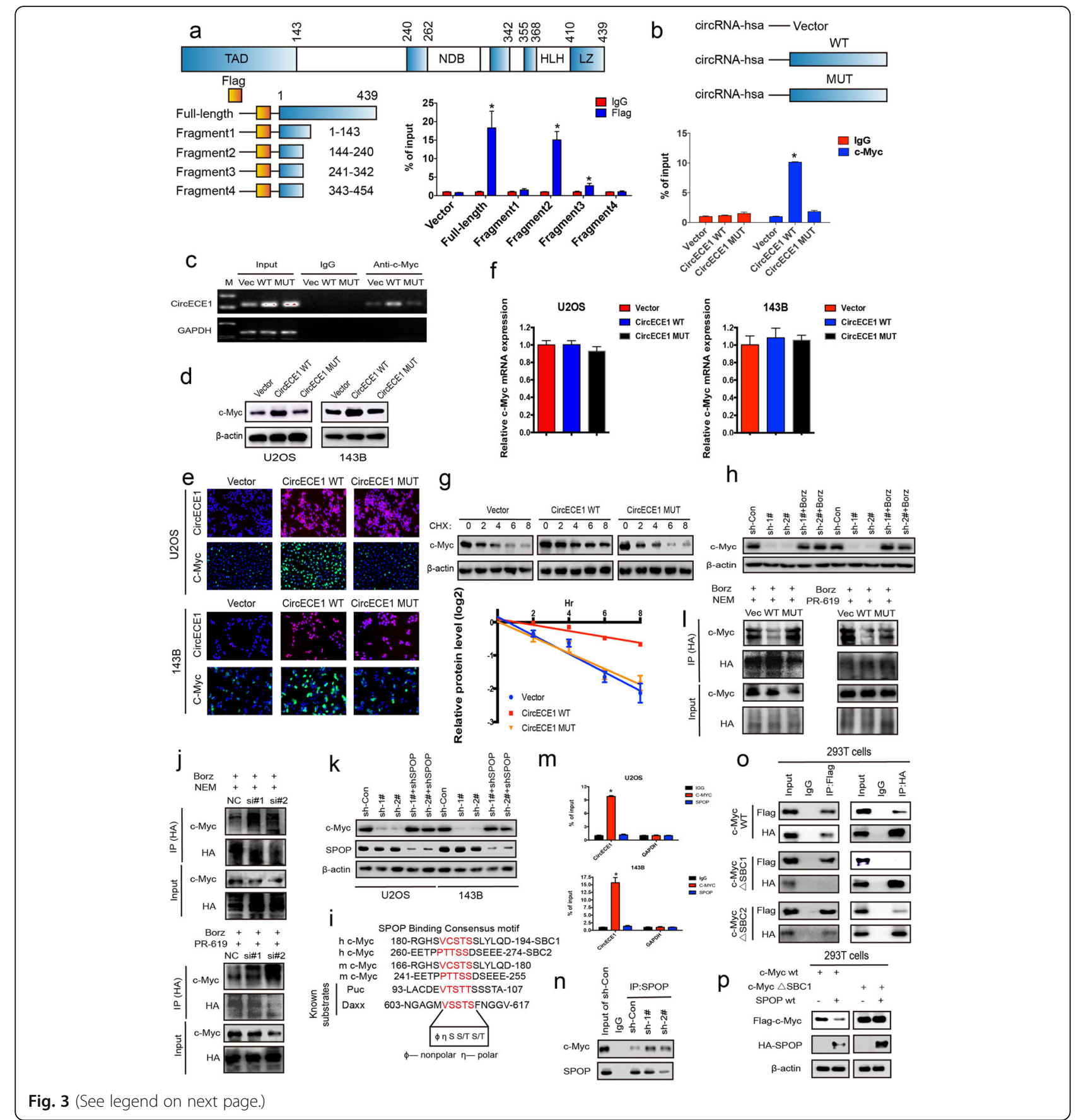

T S/T ( $\phi$-nonpolar; $\pi$-polar). Interestingly, c-Myc has two putative SBC sequences (SBC1: aa 185 VCSTS 189 and SBC2: aa 261 PTTSS 265). Further, SBC1 is located in the CircECE1 binding site (Fig. 31). We therefore hypothesized that CircECE1 may compete with SPOP to block its interaction with c-Myc, thus preventing SPOPmediated degradation. We first performed a RIP assay, which showed that CircECE1 interacts with $\mathrm{c}-\mathrm{Myc}$, but not SPOP (Fig. 3m). Next, we performed Co-IP experiments, and CircECE1 knockdown enhanced the interaction between SPOP and c-Myc in 143B cells (Fig. 3n). Notably, while WT c-Myc can bind to the HAtagged SPOP, c-Myc mutated at SBC1 ( $\triangle \mathrm{SBC} 1)$ was unable to bind to SPOP (Fig. 3o). Mutation at SBC2 $(\triangle \mathrm{SBC} 2)$ also impaired the SPOP-c-Myc interaction, but residual binding persisted (Fig. $3 \mathrm{p}$ ). These data suggest that SBC1 is the major SPOP binding site, whereas SBC2 plays a dispensable role in SPOP-c-Myc binding. All these results indicate that CircECE1 interacts with $\mathrm{c}$ Myc to prevent SPOP-mediated degradation. 
(See figure on previous page.)

Fig. 3 CircECE1 interacts with c-Myc to prevent SPOP-mediated degradation. a. Identification of the regions of the c-Myc protein that interact with CircECE1. The fragments of the c-Myc protein are illustrated (left); the interaction of c-Myc protein regions with CircECE1 in 293 T cells was confirmed using a RIP assay (right). The interaction of CircECE1 with c-Myc and SPOP was verified using a RIP assay. b-c. Schematic diagram of CircECE1 WT and MUT (left); RNA immunoprecipitation experiments were performed using anti-c-Myc antibodies in 143B cells by qRT-PCR (b) and RT-PCR(c), and specific primers were used to detect CircECE1 or GAPDH; $n=3$. d-e. The effect of CircECE1 WT/MUT on the expression of c-Myc protein levels in OS cells as assessed by WB (d) and IF (e). $\mathbf{f}$. The effect of CircECE1 WT/MUT on the expression of c-Myc mRNA levels in OS cells as assessed by qRT-PCR. $\mathbf{g}$. The effect of CHX treatment on the change in the c-Myc protein level mediated by CircECE1 WT and MUT overexpression as detected by western blotting. $\mathbf{h}$. The effect of Bortezomib treatment on the change in the c-Myc protein level mediated by CircECE1 knockdown as detected by western blotting. i. The ubiquitination levels of c-Myc in CircECE1 WT or MUT cells as measured by IP experiments. Bortezomib (250 nM) and NEM (5 $\mu \mathrm{m}$ )(Protease inhibitor) or PR-619 (100 nm)(Non-selective deubiquitinating enzyme inhibitor) were added for $6 \mathrm{~h}$. $\mathbf{j}$. The ubiquitination levels of c-Myc in CircECE1 knockdown cells as measured by IP experiments. Bortezomib (250 nM) and NEM $(5 \mu \mathrm{m})$ or PR-619 $(100 \mathrm{~nm})$ were added for $6 \mathrm{~h}$. k. The effect of SPOP knockdown on the change in the c-Myc protein level mediated by CircECE1 knockdown as detected by western blotting. I. Sequence alignment of c-Myc with the SPOP binding motif (SBC) in known SPOP substrates. $\mathbf{m}$. The interaction of CircECE1 WT and MUT with c-Myc in OS cells was verified using a RIP assay. $\mathbf{n}$. The interaction of c-Myc with SPOP was verified using a Co-IP assay. o. Role of SBC1 and SBC2 in the direct interaction of SPOP with c-Myc. 293 T cells were transfected with expression vectors for SPOP WT and FLAG- c-Myc or with c-Myc that was mutated within the SBC $(\triangle \mathrm{SBC}) 1$ or 2 for $24 \mathrm{~h}$. Bortezomib (250 nM) was then added for 6 h. Total cell lysates were prepared, and IP experiment was conducted using anti-FLAG or anti-SPOP antibodies. p. 293 T cells were transfected as indicated with HA-SPOP WT and Flag-c-Myc or Flag-c-Myc $\triangle \mathrm{SBC1}$ for $24 \mathrm{~h}$. Immunoblot analyses were conducted to detect for Flag-tagged cMyc, HA-tagged SPOP, and $\beta$-actin

\section{CircECE1 overexpression promotes the Warburg effect} It has been well documented that c-Myc promotes glycolysis through upregulation of various target genes. Therefore, we investigated whether CircECE1 is involved in glycolysis and energy metabolism. To further study the potential function of CircECE1 in the regulation of glucose metabolism, we examined whether CircECE1 overexpression or knockdown affected the expression of a panel of genes involved in glucose metabolism. qPCR and WB analyses revealed that CircECE1 dysregulation affected the expression levels of various key regulators involved in glucose uptake, glycolysis, and lactate secretion (Fig. 4a-c). Specifically, CircECE1 overexpression increased the levels of several glycolysis genes, including Glut1, Glut4, hexokinase 2, and the lactate transporter MCT4 (Fig. 4b-c). CircECE1 overexpression also increased the expression levels of pyruvate dehydrogenase (PDK) 1 and PDK4 (Fig. 4b-c), whereas CircECE1 knockdown had the opposite effect. To meet the demands of rapid proliferation and metastasis, malignant tumor cells rely on glucose metabolism for ATP production. Therefore, we analyzed the impact of CircECE1 on ATP production. CircECE1 overexpression increased ATP production in U2OS and 143B cells, whereas CircECE1 knockdown decreased ATP production (Fig. 4d). The mitochondrial membrane potential, which is used to evaluate early apoptosis, reflects mitochondrial integrity and varies according to the metabolic state. CircECE1 increased the mitochondrial membrane potential in U2OS and 143B cells (Fig. 4e), indicating that CircECE1 also functions as a positive regulator of mitochondrial glucose metabolism. Correspondingly, CircECE1 knockdown led to decreased glucose uptake and lactate production
(Fig. 4f-g). Seahorse analysis also indicated an increased extracellular acidification rate (ECAR) and oxygen consumption rate (OCR) in CircECE1overexpressing cells (Fig. 4h). Taken together, our results revealed that CircECE1 plays a vital role in glucose metabolism in OS cells.

\section{C-Myc mediates the functions of CircECE1 in glucose metabolism}

We next investigated whether c-Myc was required for the role of CircECE1 in glucose metabolism. The expression of glycolytic enzymes related to glucose metabolism could not be upregulated after c-Myc was knocked down (Fig. 5a-b). Further, ATP production decreased with CircECE1 MUT overexpression compared to CircECE1 WT overexpression (Fig. 5c). CircECE1 MUT also decreased the mitochondrial membrane potential of U2OS and 143B cells compared to CircECE1 WT (Fig. 5d). Subsequent analysis indicated that CircECE1 MUT inhibited glucose uptake and lactate production compared to CircECE1 WT in U2OS and 143B cells (Fig. 5e). The ECAR and OCR results measured using a Seahorse metabolism analyzer further confirmed that CircECE1 MUT did not have an effect on glycolysis and mitochondrial respiration, but CircECE1 WT did (Fig. 5f). These results confirm that c-Myc mediates the functions of CircECE1 in glucose metabolism.

We then conducted CCK8, EDU, and colony formation assays, which demonstrated that CircECE1 MUT significantly suppressed the proliferation and colony formation ability of OS cells compared to CircECE1 WT (Fig. 5g-i). Additionally, the migration abilities of OS cells were prominently decreased by CircECE1 MUT compared to CircECE1 WT (Fig. 5j). Moreover, CircECE1 MUT augmented the apoptosis rate 
a
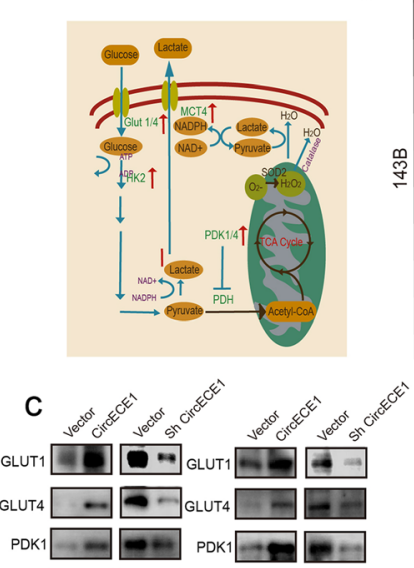

PDK4 $-6, \quad$ PDK4 $-\infty$
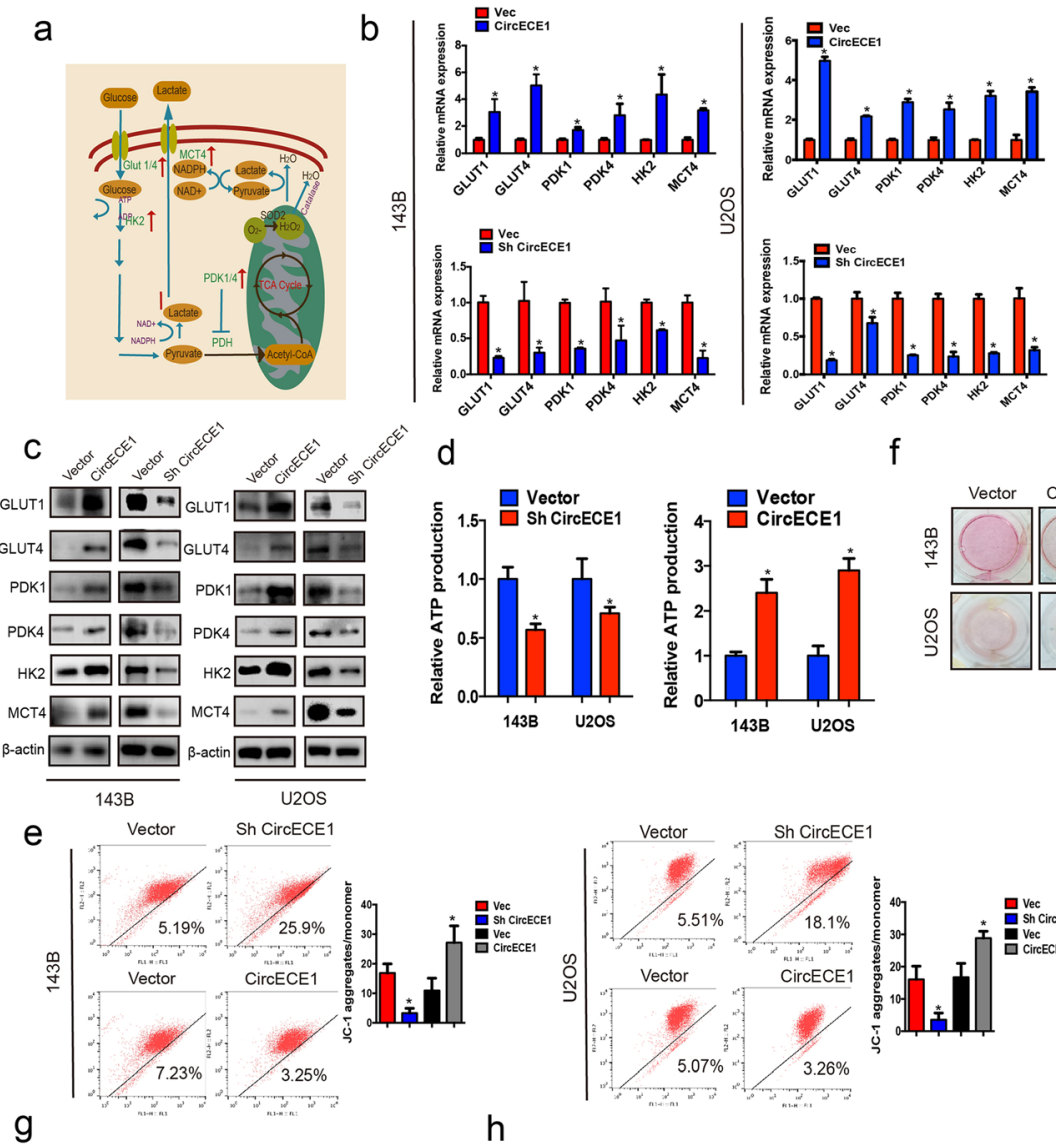

$f$
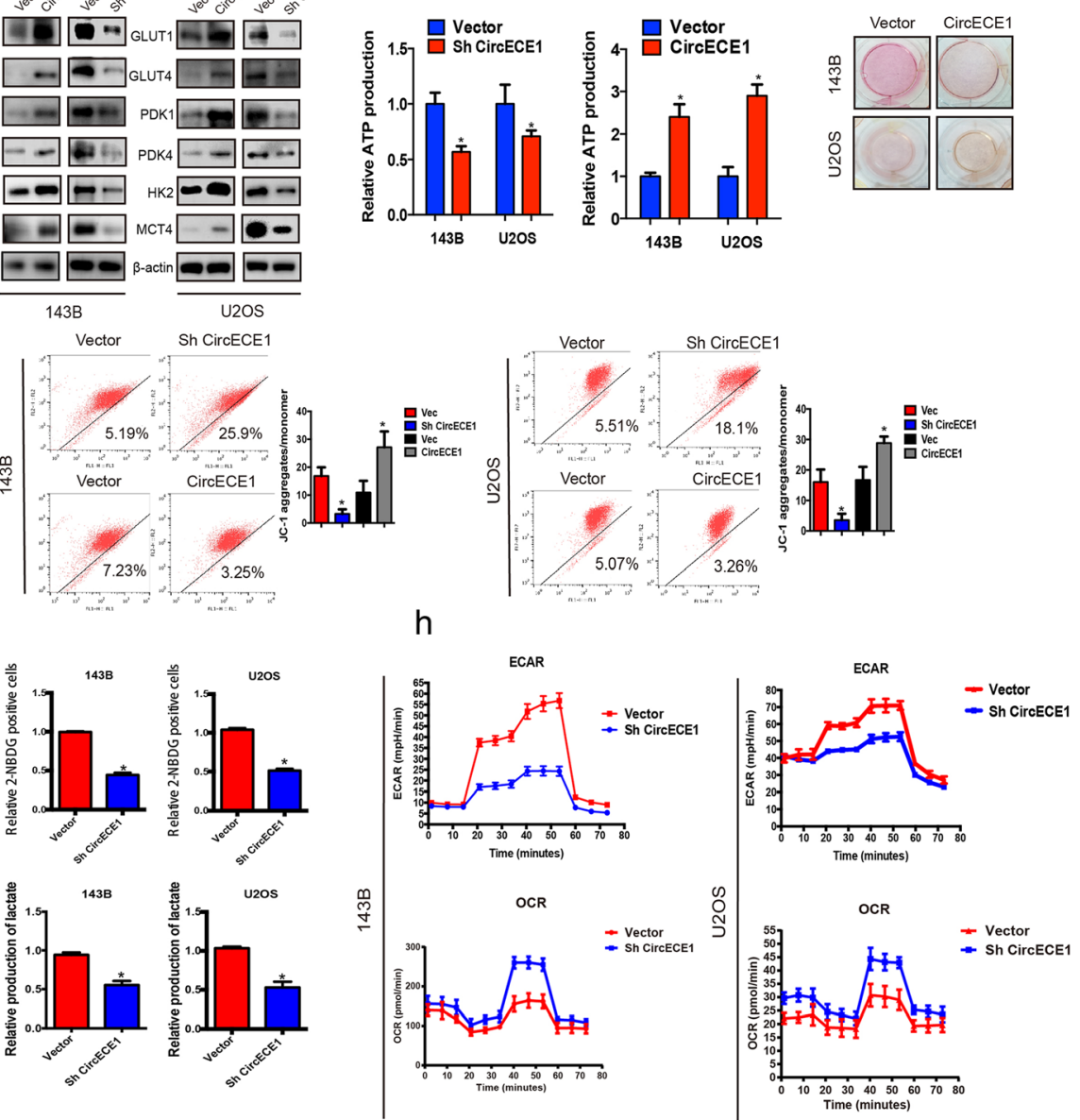

Fig. 4 CircECE1 is a positive regulator of glucose metabolism in osteosarcoma. a. Schematic representation of glucose metabolism in cancer cells. b-c. OS cells infected with either control shRNA or CircECE1 shRNA were subjected to various analyses to measure the expression levels of genes involved in glucose metabolism by real-time PCR (b) or WB (c). OS cells infected with either control vector or CircECE1 overexpression vector were subjected to various analyses to measure the expression levels of genes involved in glucose metabolism by real-time PCR (b) or WB (c). d. CircECE1 overexpression increased ATP production and CircECE1 knockdown decreased ATP production. e. Mitochondrial potential increased in the presence of CircECE1 overexpression and decreased in the presence of CircECE1 knockdown. f. CircECE1 induced lactate production as indicated by the color of the medium. g. CircECE1 downregulation inhibited glucose uptake and lactate production in osteosarcoma cells. $\mathbf{h}$. Extracellular acidification rate (ECAR), an indicator of glycolysis, was reduced in response to CircECE1 knockdown. Oxygen consumption rate (OCR), which reflects mitochondrial respiration, was decreased in CircECE1 inhibited osteosarcoma cells

compared to CircECE1 WT (Fig. 5k). Taken together, these findings indicate that $\mathrm{c}-\mathrm{Myc}$ mediates the functions of CircECE1 in glucose metabolism.
TXNIP is a target of CirCECE1 in OS

To identify the molecular mechanism underlying the CircECE1-mediated regulation of glucose metabolism, 


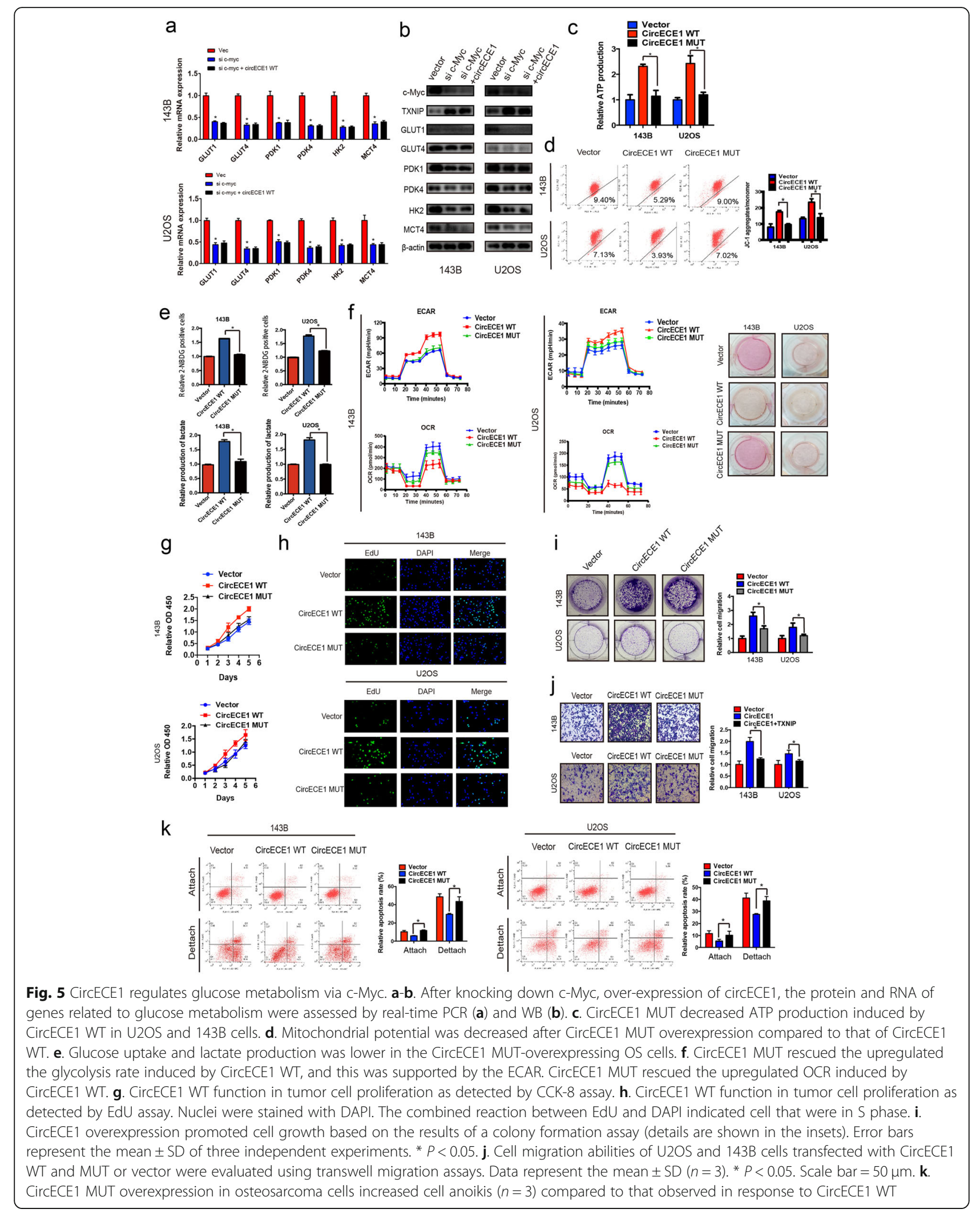


we performed RNA-seq and found a series of signaling pathways altered by CircECE1 overexpression (Fig. 6a). TXNIP, one of the differentially expressed genes, is a well-established regulator of glucose metabolism and is regulated by c-Myc in some cancer cells [24-26]. We first explored whether TXNIP was also regulated by cMyc in OS and examined its function in OS. Both cMyc and CircECE1 knockdown significantly altered TXNIP mRNA expression in two OS cell lines (Fig. 6b). Additionally, c-Myc inhibited TXNIP promoter activity in a luciferase assay (Fig. 6c). Moreover, c-Myc occupied the E-boxes in the TXNIP promoter region, as determined by a chromatin immunoprecipitation assay (Fig. 6d), and c-Myc knockdown increased TXNIP protein levels in OS cells (Fig. 6e \& Fig. S6a). These findings suggest that c-Myc blocks TXNIP transcription.

We then generated stable U2OS and 143B cell lines with ectopic TXNIP overexpression or with TXNIP knockdown via shRNA (Fig. S6b). A subsequent analysis indicated that TXNIP inhibited the expression of glycolytic enzymes related to glucose metabolism (Fig. 6f). We also examined the influence of TXNIP on OS cell proliferation. As expected, a CCK8 proliferation assay indicated that TXNIP decreased the proliferation of U2OS and 143B cells (Fig. 6g). Colony formation assays demonstrated that TXNIP also inhibited the colony formation ability of OS cells (Fig. 6h).

We hypothesized that TXNIP was a potential effector of CircECE1 in the regulation of glucose metabolism. To test this hypothesis, we examined the expression of TXNIP in two CircECE1-overexpressing OS cell lines. TXNIP protein levels decreased following CircECE1 overexpression (Fig. 6i). Further, we overexpressed TXNIP in CircECE1 WT U2OS and 143B cells and found that TXNIP could reverse the effects of CircECE1 WT overexpression in vitro, including regulating the expression of various key regulators involved in glucose uptake, glycolysis, lactate secretion, ATP production, glucose uptake, lactate production, ECAR, OCR, proliferation, migration, and anoikis (Fig. 6i-r). Therefore, we believe that TXNIP is an important downstream effector of CircECE1 in regulating glucose metabolism.

\section{CirCECE1 promotes tumor growth and metastasis and increases glucose utilization in a xenograft model}

To further confirm the role of CircECE1 in glucose metabolism, we subcutaneously injected nude mice with CircECE1 WT- or MUT-overexpressing 143B cells. As expected, compared with the control or CircECE1 MUT cells, CircECE1 WT cells had a higher tumor growth rate (Fig.7a). We observed similar results for the average tumor wet weight and tumor volume in the three groups (Fig. 7b-c). Subsequent WB, RT-qPCR, and immunohistochemistry analysis using antibodies against GLUT1,
GLUT4, PDK1/4, and MCT4 indicated that the mRNA and protein levels of these glycolytic enzymes were significantly upregulated in tissues from CircECE1 WT group xenograft tumors (Fig. 7d-f \& Fig. S7), which was consistent with our in vitro results. Considering that OS tends to metastasize, we injected 143B cells labeled with both a luminescent dye and GFP into the tail vein for seeding into the lung cavity. In vivo bioluminescence imaging demonstrated that CircECE1 WT promoted the metastasis of OS cells in situ, whereas CircECE1 MUT cells were unable to form lung metastases (Fig. 7g), which is similar to the results of the subcutaneous model. Further, we measured TXNIP and glycolytic enzyme expression in chondroma and OS patient samples using IHC and found decreased TXNIP expression and increased glycolytic enzyme expression in OS (Fig. 7h). Altogether, these findings indicate that CircECE1 activates $\mathrm{C}-\mathrm{Myc}$-mediated energy metabolism by preventing its degradation by SPOP in OS (Fig. 7i).

\section{Discussion}

Preliminary studies have gradually illuminated the function and molecular mechanisms of circRNAs. circRNAs play a significant role in the development and prognosis of various cancers. Our previous study showed that CircFAT1 can act as an endogenous competitive RNA to promote OS development through regulation of the Hippo signaling pathway ${ }^{13}$. CircTADA2A accelerates OS proliferation and metastasis ${ }^{12}$, and circLARP4 acts as a sponge to regulate the expression of LARP4 by interacting with $m i R-424-5 p$ in gastric cancer [27]. Furthermore, circRNAs are involved in regulating lung cancer phenotype by serving as endogenous competitive RNAs during carcinogenesis and proliferation [27, 28]. However, few current studies have examined the specific biological function and molecular mechanism of circRNAs in OS. circRNAs, which are evolutionarily conserved in mammals, possess multiple regulatory mechanisms owing to their specific covalently closed-loop construction. Therefore, more concrete studies are urgently needed to determine the functions and molecular mechanisms of circRNAs.

In this study, we found that CircECE1 is upregulated in OS cells and OS tissues and interacts with c-Myc. We further explored the consequences of CircECE1 upregulation. CircECE1 promoted OS cell proliferation in vitro and growth in vivo. CircECE1 promoted OS cell migration in vitro and colonization in the lung in vivo. Together, these data support our conclusion that $\operatorname{CircECE1}$ has pleiotropic effects on proliferation, tumor growth, invasion, colonization, and metastasis. Therefore, we conclude that CircECE1 has oncogenic activity and promotes OS progression. 


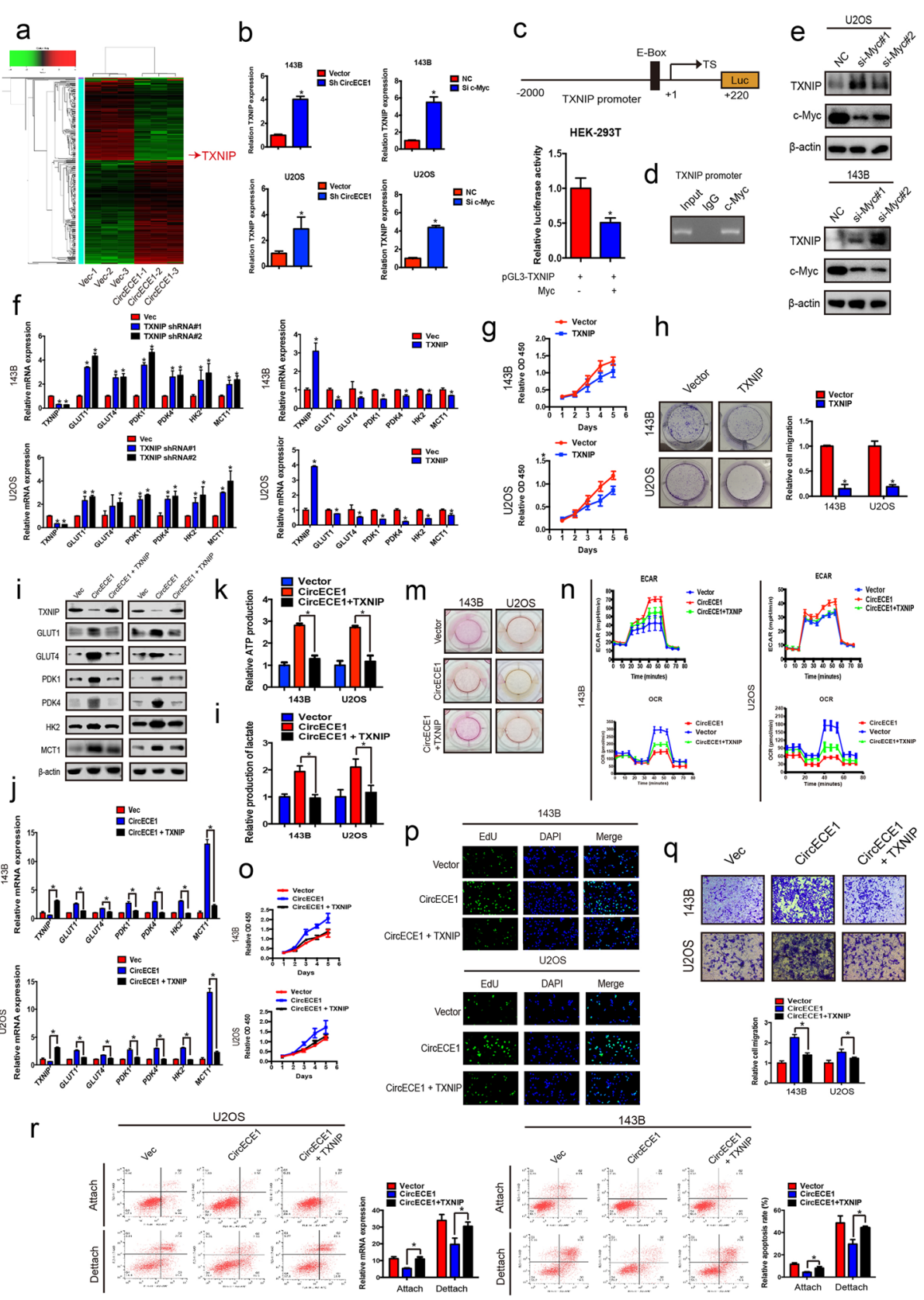

Fig. 6 TXNIP negatively regulates glucose metabolism and proliferation in osteosarcoma. a. Overexpression of CircECE1 in OS cells, and heat map detailing the RNA-seq. b. Knockdown of c-Myc or CircECE1 increased the levels of TXNIP mRNA. c. Luciferase assay showed that c-Myc binds to the TXNIP promoter. $\mathbf{d}$. c-Myc occupies the E-box of the TXNIP promoter region as measured by ChIP assay. e. Knockdown of c-Myc increased TXNIP protein expression. $\mathbf{f}$. Effect of TXNIP overexpression or knockdown on the expression levels of genes involved in glucose metabolism in OS cells as assessed by real-time PCR. $\mathbf{g}$. TXNIP inhibited cell proliferation as measured by a CCK-8 proliferation kit. $\mathbf{h}$. TXNIP inhibited cell proliferation as measured by a colony formation assay. i. Cell lysates were collected, and immunoblotting was performed using the indicated antibodies. $\mathbf{j}$. TXNIP rescued the expression levels of genes involved in glucose metabolism in OS cells by real-time PCR, and this was induced by CircECE1 overexpression. $\mathbf{k}-\mathbf{m}$. ATP and lactate production was lower in the TXNIP and CircECE1 co-expressing OS cells. $\mathbf{n}$. TXNIP and CircECE1 COexpression lowered the glycolysis rate as indicated by the ECAR and OCR. o. TXNIP and CircECE1 function in tumor cell proliferation as detected by CCK-8 assay. p. TXNIP and CircECE1 function in tumor cell proliferation as detected by EdU assay. Nuclei were stained with DAPI. A combined reaction between EdU and DAPI identified the cells in S phase. q. Cell migration abilities of U2OS and $143 \mathrm{~B}$ cells transfected with CircECE1 and TXNIP, CircECE1 alone, or vector were evaluated using transwell migration assays. Data represent the mean \pm SD $(n=3)$. * $P<0.05$. Scale bar $=$ $50 \mu \mathrm{m}$. $\mathbf{r}$. TXNIP and CircECE1 overexpression in osteosarcoma cells increased cell anoikis $(n=3)$ compared to that observed in response to CircECE1 alone 


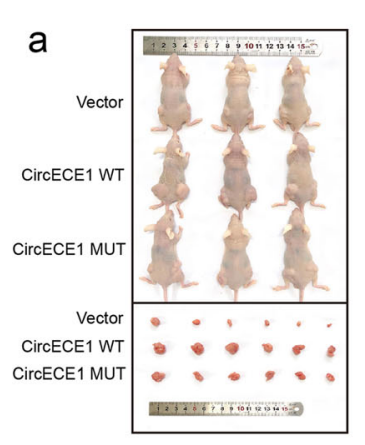

f
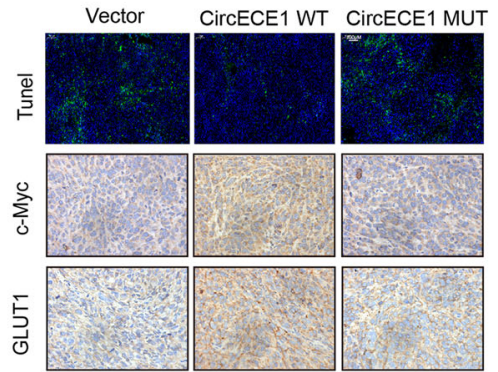

g
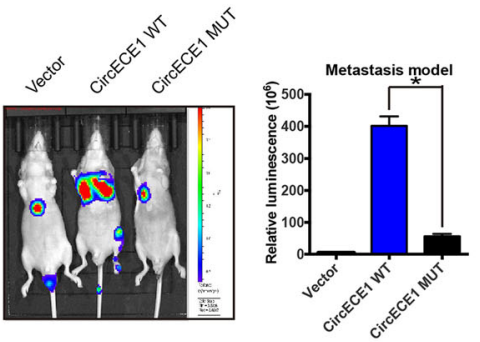

h

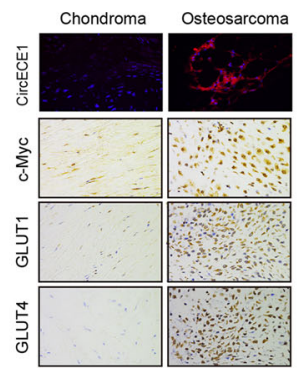

b

C
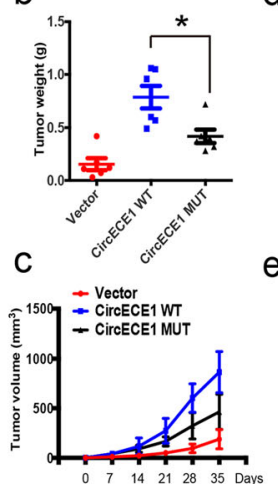

d

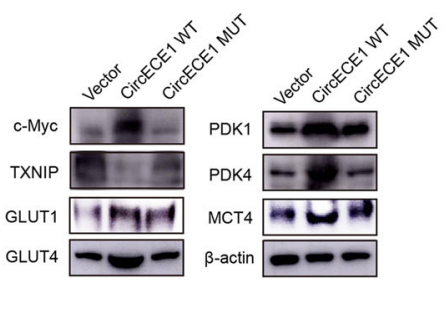

e
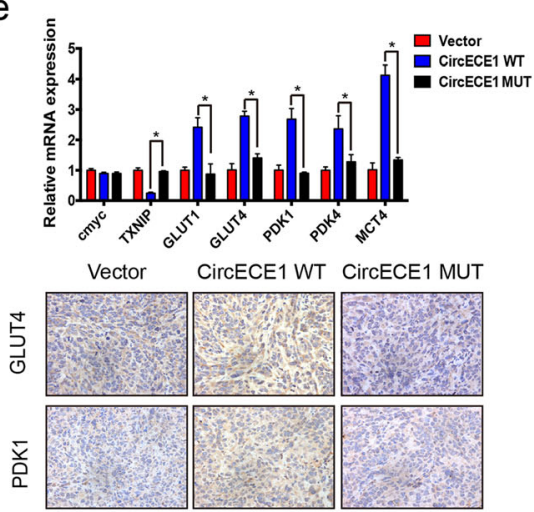

吉

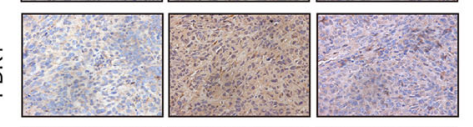

$\frac{⿱ 亠 䒑}{2}$

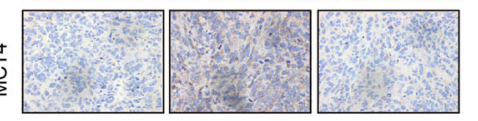

i

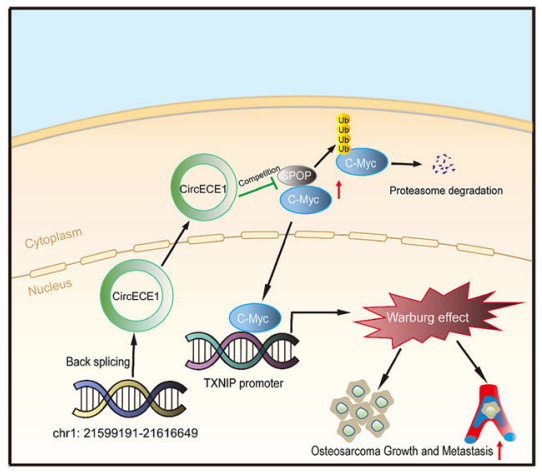

Fig. 7 CircECE1 positively regulates glucose metabolism in a xenograft model. a. 143B cells stably expressing CircECE1 WT, MUT, or empty vector were subcutaneously injected into nude mice. Nude mice were respectively injected with an equal amount of $5 \times 10^{6}$ stable control cells or cells transfected with CircECE1 WT or MUT subcutaneously. After 30 days, tumors were dissected and photographed. $\mathbf{b}$. Tumor weight was calculated on the day mice were euthanized. Data represent the mean $\pm \operatorname{SEM}(n=6$ each group). c. Tumor volumes (ab2/2) were recorded every six days beginning on the day after mice were injected with the stable OS cells. Data represent the mean \pm SEM ( $n=6$ each group). $\mathbf{d}$. Western blotting was used to assess protein expression levels. Expression of the rate-limiting enzymes GLUT1, GLUT4, MCT4, PDK1, and PDK4 was decreased in tumors formed by CircECE1 overexpression in osteosarcoma cells. e. RT-qPCR revealed that the mRNA levels of the rate-limiting enzymes GLUT1, GLUT4, MCT4, PDK1, and PDK4 were decreased in tumors formed by CircECE1 overexpression in osteosarcoma cells. $\mathbf{f}$. H\&E staining and immunohistochemistry (IHC) revealed the structure of OS in mice and the relative protein levels of GLUT1, GLUT4, MCT4, PDK1, and PDK4 in tumors of different groups. Scale bars $=100 \mu \mathrm{m}$. g. Lung metastasis in mice injected with various stable $143 B$ cells in the tail vein was detected using an in vivo bioluminescence imaging system. Representative images and a histogram are shown ( $n=9$ each group). $\mathbf{h}$. Fish revealed the expression level of CircECE1 and Immunohistochemistry (IHC) revealed the relative protein levels of GLUT1, GLUT4, MCT4, PDK1, and PDK4 in chondroma and osteosarcoma. Scale bars $=100 \mu \mathrm{m}$. I. Schematic illustration of the CircECE1/c-Myc/TXNIP axis 
circRNAs function as signaling pathway regulators in gene expression and other important cellular processes $[29,30]$. Many molecular functions are performed by circRNAs to implement their cellular effects, such as affecting gene cotranscription and binding to RNA or regulating protein translation by forming nuclear or cytoplasmic complexes [31-37]. Here, we demonstrated that CircECE1 knockdown decreased the levels of c-Myc protein. We further demonstrated that $\mathrm{c}-\mathrm{Myc}$ directly bound the TXNIP promoter to transcriptionally regulate TXNIP expression in OS cells. c-Myc is a crucial protooncogene in multiple malignant tumors and is closely associated with tumorigenesis, cell cycle regulation, and cell proliferation [38]. Moreover, c-Myc plays a critical role in invasion and metastasis [39]. Our previous studies showed that OS cells have higher c-Myc protein levels, indicating the presence of important c-Myc regulation mechanisms at the protein level ${ }^{13}$. Recently, Geng et al [40] found that the E3 ubiquitin ligase adapter SPOP can promote c-Myc degradation via ubiquitination. We found that the SPOP binding motif overlapped with the $\operatorname{circECE1}$ binding site. Therefore, we speculated that CircECE1 may compete with SPOP to prevent SPOP-mediated degradation. Our results indicated that CircECE1 interacts with c-Myc but not SPOP and acts to prevent SPOP-induced c-Myc degradation.

TXNIP is a glycolysis-related gene that we found to be regulated by CircECE1. We examined whether CircECE1 altered glycolysis via TXNIP in OS. Previous studies have shown that TXNIP is a cancer suppressor gene in numerous solid tumors and hematological malignancies $[41,42]$. Moreover, recent research revealed that TXNIP is a potent negative regulator of glucose uptake and aerobic glycolysis [43]. In our study, these results were confirmed by q-PCR and western blot analysis, which showed that both the mRNA and protein levels of TXNIP were remarkably higher in CircECE1 knockdown OS cells than in control cells, indicating that TXNIP was regulated by CircECE1, predominantly via transcriptional regulation.

\section{Conclusions}

Our research provides new insights into the role of circRNAs in OS and presents evidence for the molecular mechanisms by which circRNAs participate in the Warburg effect. This study identified CircECE1 as a positive regulator of glucose metabolism and showed that it plays a crucial role in OS progression. Mechanistically, CircECE1 interacts with c-Myc to prevent SPOP-induced cMyc ubiquitination and degradation and then activates c-Myc-TXNIP signaling to regulate the Warburg effect. These results may help identify new diagnostic and therapeutic targets for OS.

\section{Supplementary Information}

The online version contains supplementary material available at https://doi. org/10.1186/s12943-020-01269-4.

Additional file 1: Supplementary Figure S1. The circRNAs with high affinity to c-Myc. The ten circRNAs with high affinity to c-Myc were verified by an RIP assay. Supplementary Figure S2. The knockdown efficiency of CircECE1. A The expression levels of CircECE1 in 143B and U2OS cells after transfection of CircECE1 or control siRNAs were detected by real-time PCR. Data represents the mean $\pm S D(n=3) .{ }^{*} P<0.05$. Data represent the mean $\pm S D(n=3)$. B The expression levels of ECE1 mRNA and CircECE1 in 143B and U2OS cells after stable transfection of CircECE1 short hairpin RNAs or vector plasmids were detected by real-time PCR. Data represents the mean $\pm S D(n=3)$. ${ }^{*} P<0.05$. C The expression levels of ECE1 mRNA and CircECE1 in 143B and U2OS cells after stable transfection of CircECE1 plasmids were detected by real-time PCR. Data represents the mean $\pm S D(n=3) .{ }^{*} P<0.05$. Supplementary Figure S3. The combination of CircECE1 and c-Myc. A Prediction of the binding position of CircECE1 to the c-Myc protein (catRAPID). B Prediction of the binding sequence of CircECE1 to the c-Myc protein (CISBP-RNA). C-D Schematic diagram of CircECE1 full-length and truncated fragments $(\mathrm{C})$; The interaction of CircECE1 truncated fragments with c-Myc in 293T cells was verified by an RIP assay (D). E CircECE1 sequence labeling c-mycbinding site (red) and the mutated nucleotides (red). F The expression levels of CircECE1 in U2OS and 143B cells after stable transfection of CirCECE1 WT/MUT or vector plasmids were detected by real-time PCR. Data represents the mean $\pm S D(n=3)$. ${ }^{*} P<0.05$. Supplementary Figure S6. The knockdown efficiency of c-Myc and the overexpression efficiency of TXNIP. A The expression levels of C-Myc in U2OS and 143B cells after transfection of C-Myc or control siRNAs were detected by real-time PCR. B The expression levels of TXNIP in U2OS and 143B TXNIP OE stable cells were detected by real-time PCR. Data represents the mean \pm SD $(n=3)$. * $P<0.05$. Data represent the mean $\pm S D(n=3)$. Supplementary Figure S7. The overexpression efficiency of CircECE1 in vivo. The expression levels of CircECE1 in tumors formed by CircECE1 WT/MUT overexpression in osteosarcoma cells were detected by real-time PCR. Data represents the mean $\pm S D(n=3)$. ${ }^{*} P<0.05$.

\section{Abbreviations}

OS: Osteosarcoma; circRNAs: circular RNAs; TXNIP: Thioredoxin binding protein; HIF: Hypoxia-inducible factor; RIP: RNA immunoprecipitation; shRNA: Small hairpin RNA; FISH: RNA fluorescence in situ hybridization; SPOP: Speckle-type POZ; SBC: SPOP binding motif; qRT-PCR: Quantitative real-time polymerase chain reaction

\section{Acknowledgements}

Not applicable.

\section{Authors' contributions}

S.S. and T.Y. contributed equally to this work. S.S., S.F. and J.M. designed the research. S.S., T.Y., Y.X., D.Z. and J.M. performed the experiments. S.S., S.F. and J.M. wrote the manuscript. All authors analyzed the data and revised the manuscript. All authors read and approved the final manuscript.

\section{Funding}

This study was supported by National Key R\&D Program of China (No. 2018YFC1105200), the Key research and development plan in Zhejiang province (No. 2018C03060), National Nature Science Fund of China (81802680), National Nature Science Fund of China (81972504) No benefits in any form have been or will be received from a commercial party related directly or indirectly to the subject of this manuscript.

\section{Availability of data and materials}

The datasets used and/or analysed during the current study are available from the corresponding author on reasonable request.

\section{Ethics approval and consent to participate}

The present study was approved by the Ethics Committee of Sir Run Run Shaw Hospital. 


\section{Consent for publication}

Obtained.

\section{Competing interests}

The authors declare no conflict of interest.

\section{Author details}

${ }^{1}$ Department of Orthopaedic Surgery, Sir Run Run Shaw Hospital, Medical College of Zhejiang University \& Key Laboratory of Musculoskeletal System Degeneration and Regeneration Translational Research of Zhejiang Province, 3 East Qingchun Road, Hangzhou 310016, Zhejiang Province, China. 2Department of Head and Neck Surgery, Institute of Micro-Invasive Surgery of Zhejiang University, Sir Run Run Shaw Hospital, Medical College of Zhejiang University, 3 East Qingchun Road, Hangzhou 310016, Zhejiang Province, China.

Received: 31 March 2020 Accepted: 16 October 2020 Published online: 26 October 2020

\section{References}

1. Ma O, Cai WW, Zender L, Dayaram T, Shen J, Herron AJ, Lowe SW, Man TK Lau CC, Donehower LA. MMP13, Birc2 (CIAP1), and Birc3 (CIAP2), amplified on chromosome 9, collaborate with p53 deficiency in mouse osteosarcoma progression. Cancer Res. 2009;69:2559-67.

2. Kaste SC, Pratt CB, Cain AM, Jones-Wallace DJ, Rao BN. Metastases detected at the time of diagnosis of primary pediatric extremity osteosarcoma at diagnosis: imaging features. Cancer. 1999;86:1602-8.

3. Warburg O. On the origin of cancer cells. Science. 1956;123:309-14.

4. Hansen TB, Jensen TI, Clausen BH, Bramsen JB, Finsen B, Damgaard CK, Kjems J. Natural RNA circles function as efficient microRNA sponges. Nature. 2013;495:384-8.

5. Shang BQ, Li ML, Quan HY, Hou PF, Li ZW, Chu SF, Zheng JN, Bai J. Functional roles of circular RNAs during epithelial-to-mesenchymal transition. Mol Cancer. 2019;18:138.

6. Cherubini A, Barilani M, Rossi RL, Jalal MMK, Rusconi F, Buono G, Ragni E, Cantarella G, Simpson H, Peault B, Lazzari L. FOXP1 circular RNA sustains mesenchymal stem cell identity via microRNA inhibition. Nucleic Acids Res. 2019:47:5325-40.

7. Nan A, Chen L, Zhang N, Jia Y, Li X, Zhou H, Ling Y, Wang Z, Yang C, Liu S, Jiang Y. Circular RNA circNOL10 inhibits lung Cancer development by promoting SCLM1-mediated transcriptional regulation of the Humanin polypeptide family. Adv Sci (Weinh). 2019;6:1800654

8. Wesselhoeft RA, Kowalski PS, Anderson DG. Engineering circular RNA for potent and stable translation in eukaryotic cells. Nat Commun. 2018;9:2629.

9. Liang WC, Wong CW, Liang PP, Shi M, Cao Y, Rao ST, Tsui SK, Waye MM, Zhang Q, Fu WM, Zhang JF. Translation of the circular RNA circbeta-catenin promotes liver cancer cell growth through activation of the Wnt pathway. Genome Biol. 2019;20:84

10. Zhong Y, Du Y, Yang X, Mo Y, Fan C, Xiong F, Ren D, Ye X, Li C, Wang Y, et al. Circular RNAs function as ceRNAs to regulate and control human cancer progression. Mol Cancer. 2018;17:79.

11. Chen J, Liu G, Wu Y, Ma J, Wu H, Xie Z, Chen S, Yang Y, Wang S, Shen P, et al. CircMYO10 promotes osteosarcoma progression by regulating miR370-3p/RUVBL1 axis to enhance the transcriptional activity of beta-catenin/ LEF1 complex via effects on chromatin remodeling. Mol Cancer. 2019;18: 150.

12. Wu Y, Xie Z, Chen J, Chen J, Ni W, Ma Y, Huang K, Wang G, Wang J, Ma J, et al. Circular RNA circTADA2A promotes osteosarcoma progression and metastasis by sponging miR-203a-3p and regulating CREB3 expression. Mol Cancer. 2019:18:73.

13. Liu G, Huang K, Jie Z, Wu Y, Chen J, Chen Z, Fang X, Shen S. CircFAT1 sponges miR-375 to promote the expression of yes-associated protein 1 in osteosarcoma cells. Mol Cancer. 2018:17:170.

14. Terry S, Faouzi Zaarour R, Hassan Venkatesh G, Francis A, El-Sayed W, Buart S, Bravo P, Thiery J, Chouaib S. Role of hypoxic stress in regulating tumor immunogenicity, resistance and plasticity. Int J Mol Sci. 2018;19.

15. Meng W, Hao Y, He C, Li L, Zhu G. Exosome-orchestrated hypoxic tumor microenvironment. Mol Cancer. 2019;18:57.

16. Vander Heiden MG, DeBerardinis RJ. Understanding the intersections between metabolism and Cancer biology. Cell. 2017;168:657-69.
17. Porporato PE, Filigheddu N, Pedro JMB, Kroemer G, Galluzzi L. Mitochondrial metabolism and cancer. Cell Res. 2018:28:265-80.

18. Hsu PP, Sabatini DM. Cancer cell metabolism: Warburg and beyond. Cell. 2008;134:703-7.

19. Xiang $S$, Gu H, Jin L, Thorne RF, Zhang XD, Wu M. LncRNA IDH1-AS1 links the functions of c-Myc and HIF1alpha via IDH1 to regulate the Warburg effect. Proc Natl Acad Sci U S A. 2018;115:E1465-e1474.

20. Sanderson SM, Locasale JW. Revisiting the Warburg effect: some tumors hold their breath. Cell Metab. 2018;28:669-70.

21. Kelly B, O'Neill LA. Metabolic reprogramming in macrophages and dendritic cells in innate immunity. Cell Res. 2015;25:771-84.

22. Masui K, Tanaka K, Akhavan D, Babic I, Gini B, Matsutani T, Iwanami A, Liu F, Villa GR, Gu Y, et al. mTOR complex 2 controls glycolytic metabolism in glioblastoma through FoxO acetylation and upregulation of c-Myc. Cell Metab. 2013:18:726-39.

23. Dang CV, Le A, Gao P. MYC-induced cancer cell energy metabolism and therapeutic opportunities. Clin Cancer Res. 2009:15:6479-83.

24. Ji S, Qin Y, Liang C, Huang R, Shi S, Liu J, Jin K, Liang D, Xu W, Zhang B, et al. FBW7 (F-box and WD repeat domain-containing 7) negatively regulates glucose metabolism by targeting the c-Myc/TXNIP (Thioredoxin-binding protein) Axis in pancreatic Cancer. Clin Cancer Res. 2016;22:3950-60.

25. Shen L, O'Shea JM, Kaadige MR, Cunha S, Wilde BR, Cohen AL, Welm AL, Ayer DE. Metabolic reprogramming in triple-negative breast cancer through Myc suppression of TXNIP. Proc Natl Acad Sci U S A. 2015;112:5425-30.

26. Zhang B, Lyu J, Liu Y, Wu C, Yang EJ, Pardeshi L, Tan K, Wong KH, Chen Q, Xu X, et al. BRCA1 deficiency sensitizes breast cancer cells to bromodomain and extra-terminal domain (BET) inhibition. Oncogene. 2018;37:6341-56.

27. Zhang J, Liu H, Hou L, Wang G, Zhang R, Huang Y, Chen X, Zhu J. Circular RNA LARP4 inhibits cell proliferation and invasion of gastric cancer by sponging miR424-5p and regulating LATS1 expression. Mol Cancer. 2017:16:151.

28. Wang L, Tong X, Zhou Z, Wang S, Lei Z, Zhang T, Liu Z, Zeng Y, Li C, Zhao J, et al. Circular RNA hsa_circ 0008305 (circPTK2) inhibits TGF-beta-induced epithelial-mesenchymal transition and metastasis by controlling TIF1gamma in non-small cell lung cancer. Mol Cancer. 2018:17:140.

29. Hu W, Bi ZY, Chen ZL, Liu C, Li LL, Zhang F, Zhou Q, Zhu W, Song YY, Zhan BT, et al. Emerging landscape of circular RNAs in lung cancer. Cancer Lett. 2018:427:18-27.

30. Kristensen LS, Hansen TB, Veno MT, Kjems J. Circular RNAs in cancer: opportunities and challenges in the field. Oncogene. 2018;37:555-65.

31. Zhang X, Xu Y, Qian Z, Zheng W, Wu Q, Chen Y, Zhu G, Liu Y, Bian Z, Xu W, et al. circRNA_104075 stimulates YAP-dependent tumorigenesis through the regulation of HNF4a and may serve as a diagnostic marker in hepatocellular carcinoma. Cell Death Dis. 2018:9:1091.

32. Ashwal-Fluss R, Meyer M, Pamudurti NR, Ivanov A, Bartok O, Hanan M, Evantal N, Memczak S, Rajewsky N. Kadener S: circRNA biogenesis competes with pre-mRNA splicing. Mol Cell. 2014;56:55-66

33. Du WW, Zhang C, Yang W, Yong T, Awan FM, Yang BB. Identifying and characterizing circRNA-protein interaction. Theranostics. 2017;7:4183-91.

34. Li X, Yang L, Chen LL. The biogenesis, functions, and challenges of circular RNAs. Mol Cell. 2018;71:428-42.

35. Qu S, Yang X, Li X, Wang J, Gao Y, Shang R, Sun W, Dou K, Li H. Circular RNA: a new star of noncoding RNAs. Cancer Lett. 2015;365:141-8.

36. Patop IL, Wust $\mathrm{S}$, Kadener $\mathrm{S}$. Past, present, and future of circRNAs. EMBO J. 2019;38:e100836.

37. Dang CV. MYC on the path to cancer. Cell. 2012:149:22-35.

38. Hessmann E, Schneider G, Ellenrieder V, Siveke JT. MYC in pancreatic cancer: novel mechanistic insights and their translation into therapeutic strategies. Oncogene. 2016;35:1609-18

39. Gupta A, Ajith A, Singh S, Panday RK, Samaiya A, Shukla S. PAK2-c-MycPKM2 axis plays an essential role in head and neck oncogenesis via regulating Warburg effect. Cell Death Dis. 2018;9:825.

40. Geng C, Kaochar S, Li M, Rajapakshe K, Fiskus W, Dong J, Foley C, Dong B, Zhang L, Kwon OJ, et al. SPOP regulates prostate epithelial cell proliferation and promotes ubiquitination and turnover of c-MYC oncoprotein. Oncogene. 2017:36:4767-77

41. Morrison JA, Pike LA, Sams SB, Sharma V, Zhou Q, Severson JJ, Tan AC, Wood WM, Haugen BR. Thioredoxin interacting protein (TXNIP) is a novel tumor suppressor in thyroid cancer. Mol Cancer. 2014:13:62.

42. Zhou J, Yu Q, Chng WJ. TXNIP (VDUP-1, TBP-2): a major redox regulator commonly suppressed in cancer by epigenetic mechanisms. Int J Biochem Cell Biol. 2011;43:1668-73. 
43. Sullivan WJ, Mullen PJ, Schmid EW, Flores A, Momcilovic M, Sharpley MS, Jelinek D, Whiteley AE, Maxwell MB, Wilde BR, et al. Extracellular Matrix Remodeling Regulates Glucose Metabolism through TXNIP Destabilization. Cell. 2018;175:117-32 e121.

\section{Publisher's Note}

Springer Nature remains neutral with regard to jurisdictional claims in published maps and institutional affiliations.

Ready to submit your research? Choose BMC and benefit from:

- fast, convenient online submission

- thorough peer review by experienced researchers in your field

- rapid publication on acceptance

- support for research data, including large and complex data types

- gold Open Access which fosters wider collaboration and increased citations

- maximum visibility for your research: over $100 \mathrm{M}$ website views per year

At $\mathrm{BMC}$, research is always in progress.

Learn more biomedcentral.com/submissions 